\title{
O Conceito de Morte em Crianças Portadoras de Doenças Crônicas ${ }^{1}$
}

\author{
Wilma da Costa Torres ${ }^{2}$ \\ Universidade Federal do Rio de Janeiro
}

\begin{abstract}
RESUM0: O estudo investiga a relação entre o nível cognitivo e o conceito de morte em crianças portadoras de doenças crônicas e compara a evolução deste conceito entre essas crianças e crianças sadias do mesmo nível cognitivo e mesmo nível sócio econômico (carência). Foram empregadas tarefas piagetianas para a avaliação do nível cognitivo e o Instrumento de Sondagem do Conceito de Morte (Torres, 1979) para avaliar o conceito de morte-biológica. A amostra foi constituída de 167 crianças portadoras de doenças crônicas e 142 crianças sadias já testadas em estudo precedente (Torres, 1996). Os resultados revelaram que a defasagem cognitiva, encontrada em crianças carentes, em relação aos padrões piagetianos não está agravada nas crianças doentes. A doença crônica embora se manifeste inicialmente como um fator desestruturante na aquisição do conceito de morte (fase pré-operacional), surge na fase seguinte (operacional-concreta) como um fator de amadurecimento na compreensão deste conceito.
\end{abstract}

Palavras-chave: criança; conceito de morte; doença crônica

\section{The Concept of Death in Children With Chronic Diseases}

\begin{abstract}
This study investigates the relationship between cognitive level and the concept of death in children with chronic diseases and compares the evolution of this concept between these children and healthy ones with the same cognitive level and the same socio economics background (poor). Cognitive level was avaliated through piagetian tasks and the Probing Tool for Concept of Death (Torres, 1979), measured the concept of biologic death. The sample included 167 chronic diseased children and 142 healthy children that had been tested in a previous study (Torres, 1996). The results showed that cognitive level of deprived children according piagetian standards is not worst among diseased children. Although chronic diseases initially appears as a deconstructive factor for the acquisition of the concept of death (pre-operational phase), in the following phase (operational-concrete) it appears as a factor conducing to a maturer understading of this concept.
\end{abstract}

Key words: child; concept of death; chronic disease

As investigações sobre a compreensão da morte pela criança, que se iniciaram com o trabalho pioneiro de Schilder e Wechsler (1934) e prosseguiram com os estudos de Nagy (1959), começaram a crescer na década de 60, e se intensificaram nas décadas de 70 e 80 , chegando atualmente a fornecer uma ampla gama de informações sobre este tópico.

Embora se observe diferenças na forma como os diversos pesquisadores enfocam o conceito de morte, alguns estudiosos (Kane, 1979; Speece \& Brent, 1984; Torres, 1979) assinalaram a importância de que o mesmo seja investigado não como um conceito único, mas como um conceito complexo, multidimensional, que envolve subconceitos, sendo a universalidade, a não-funcionalidade e a irreversibilidade os três componentes mais amplamente pesquisados.

A pesquisa de Nagy (1959) que representa o ponto de partida para examinar como as crianças conceituam a morte

1 Pesquisa desenvolvida com o apoio financeiro do CNPq sob a forma de bolsa de pesquisa para a autora e bolsas de Iniciação Científica para alunos participantes: Anriet Barros de Siqueira, Christianne Ramos M. Barbosa, Felipe F. Garcia, Mira Carla P. de Noronha. Além dos bolsistas, colaboraram com a pesquisa: Ana Cláudia Coelho de Oliveira e Manuel Gil. Resultados parciais deste trabalho foram apresentados pelos bolsistas em sessão de Tema Livre no VIII Encontro Nacional dos Psicólogos da Área Hospitalar, Curitiba, Paraná, 1999.

2 Endereço: Núcleo de Estudos e Pesquisas em Tanatologia do Instituto de Psicologia da UFRJ - Av. Pasteur 250, CEP: 22295-900, Rio de Janeiro, RJ - E-mail: wilmatorres@zipmail.com.br em diferentes idades, assim como os estudos que se seguiram (Bolduc, 1972; Tallmer, Formanek \& Tallmer, 1974; Weininger, 1979), apontam para o fato de que as crianças nas fases iniciais do desenvolvimento freqüentemente pensam sobre a morte como reversível, atribuem funções definidoras da vida às coisas mortas e não acreditam na inevitabilidade da morte. No que se refere à idade, a maioria das pesquisas sugere que a aquisição dos três componentes irreversibilidade, não-funcionalidade e universalidade - situa-se em torno dos 7 anos (Speece \& Brent, 1984).

Como a compreensão da morte na criança não se faz independentemente do desenvolvimento cognitivo global, além da idade, esta tem sido a outra variável mais empregada na investigação sobre a aquisição do conceito de morte. Kastenbaum e Aisemberg (1976/1983) forneceram os fundamentos lógicos da relação entre o conceito de morte e a maturação da cognição. Também Koocher (1972) assinala que a perspectiva mais lógica para iniciar a investigação sobre este conceito é ligando-o à estrutura do desenvolvimento cognitivo, uma vez que a compreensão da morte ou de qualquer outro fenômeno natural está limitada pela capacidade com que a criança é capaz de perceber, interpretar, classificar e integrar o estímulo de seu ambiente.

Entre as pesquisas que focalizaram o conceito de morte em função do desenvolvimento cognitivo, algumas (Anthony, 1972; Kane, 1978, 1979; Safier, 1964) concluíram pelo desenvolvimento de uma compreensão da morte paralela às 
etapas de Piaget, sem manifestarem uma preocupação com os modos específicos pelos quais a capacidade de conceitualizar os diferentes componentes do conceito de morte se relaciona com o desenvolvimento cognitivo. Outro grupo de pesquisas procurou especificar as relações lógicas a nível mais microscópico, sugerindo certas aptidões - classificação, compreensão linear de tempo, reciprocidade - como sendo as realizações cognitivas específicas essenciais à compreensão dos três componentes do conceito de morte (Hornblum, 1978; Kalmbach, 1979; Koocher, 1972; Townley \& Thornburg, 1980; Torres, 1979).

Da mesma forma que as pesquisas que focalizam a aquisição do conceito de morte em função da idade não são conclusivas, também aquelas que focalizam esta aquisição em função do nível cognitivo não o são. Entre as razões apontadas para a inconsistência desses resultados pode-se destacar: aquelas inerentes a toda pesquisa sobre desenvolvimento, a de investigar processos longitudinais, como a evolução de conceitos, através de dados obtidos em estudos transversais; as de ordem instrumental, ou dos procedimentos, para avaliar a complexidade dos diferentes componentes do conceito de morte e o nível cognitivo da criança; por último, as dificuldades de ordem teórica na tentativa de determinar as implicações da teoria geral do desenvolvimento cognitivo de Piaget com o desenvolvimento específico de conceitos abstratos como o conceito de morte (Bibace \& Walsh, 1980; ver também Spence \& Brent, 1984). Na maioria das pesquisas, apesar da relação encontrada entre o desenvolvimento cognitivo e o conceito de morte, falta especificidade suficiente que permita explicar por que determinada etapa piagetiana é um pré-requisito para que um nível particular de compreensão de conceitos abstratos, como o de morte, seja alcançado. Falta uma descrição detalhada das aptidões específicas que estão logicamente implícitas na compreensão do conceito de morte.

Finalmente, é possível que, em princípio, a teoria de Piaget não forneça um esboço completo para o conhecimento das concepções da criança sobre a morte, uma vez que, sendo sua preocupação de natureza epistemológica, enfatiza o desenvolvimento cognitivo de contexto - independente das aptidões específicas implícitas na aquisição do conceito e das experiências da criança com a morte. Também tem sido enfatizado que a perfomance nas tarefas piagetianas freqüentemente depende do conhecimento sobre o tema ou assunto em questão (Flavell, conforme citado por Speece \& Brent, 1984). No presente caso, as diferenças nas experiências individuais da criança com a morte, e, não só as experiências, mas o que lhe é dito acerca da morte são, provavelmente, cruciais para a compreensão do conceito de morte (Speece $\&$ Brent, 1984). Aliás, a evidência atual sugere que tanto as diferenças em função da idade como as diferenças em função do nível cognitivo podem ser modeladas por variáveis tais como experiências com a morte, a tendência suicida, a doença terminal, a privação sócio-econômica, entre outras.

Com relação às experiências com a morte, embora alguns pesquisadores tenham investigado a possibilidade de que possam ter um efeito facilitador no desenvolvimento do conceito de morte mais realista (Bolduc, 1972; Cotton \& Range, 1990; Gartley \& Bernasconi, 1967; Kane, 1979; Peck, 1966; Reilly, Hasazi, Bond, 1983), a literatura sobre o impacto dessas experiências ainda é confusa.

Uma outra linha de investigação se refere ao impacto que a tendência suicida possa ter na aquisição do conceito de morte. Alguns pesquisadores (McIntire, Angle \& Struempler, 1972; Orbach \& Glaubman, 1978, 1979; Schilder \& Wechsler, 1934), postulam que as crianças que tentam suicídio têm conceitos de morte "distorcidos", ou "imaturos", tais como a morte como processo reversível, mais do que as crianças não suicidas. Shaffer e Fisher (1981) consideram que a crença na reversibilidade pode contribuir para o comportamento suicida. O maior problema destas pesquisas, entretanto, se refere à dificuldade para distinguir componentes afetivos dos cognitivos no que concerne à crença na reversibilidade.

Em relação à variável classe social, observa-se que, de modo geral, os estudos focalizam crianças brancas, da área urbana e de classe média, podendo-se citar como exceções os estudos de McIntire e cols. (1972), Orbach e Glaubmam (1978, 1979), Shoenfeld e Smilansky (1989) e o de Tallmer e cols. (1974).

Um outro tópico importante para a investigação diz respeito ao impacto que a doença crônica grave e/ou terminal pode ter sobre a conceituação da criança acerca da morte. Em relação a estes estudos, a maioria se baseia em observações clínicas e no relato das equipes que atendem a estas crianças (Adams, 1979; Bluebond-Langner, 1974, 1978; Lonetto, 1980; Natterson \& Knudson, 1960; Raimbault, 1977/1979). Também não focalizam o conceito de morte em um sentido estrito, isto é, em termos da morte biológica, mas em termos de um conceito global que envolve tanto aspectos cognitivos quanto afetivos.

No que concerne ao peso da experiência da terminalidade, Raimbault (1977/1979) e Bluebond-Langner (1978), partindo da observação direta das crianças, não têm qualquer dúvida de que a criança sabe que vai morrer. Também autores como Natterson e Knudson (1960) e Morrisey (1963) ao concluírem que a ansiedade de separação seria a ansiedade predominante nas crianças menores, podem estar falando de ansiedade de morte, que nada mais é do que uma reativação da ansiedade do trauma do nascimento, o qual, segundo a perspectiva psicanalítica, estaria na raiz de nossas ligações conceituais com a morte. Esta é a razão pela qual Anthony (1972) enfatiza a ligação das concepções da morte na criança com o trauma do nascimento e posterior ansiedade e impulsos agressivos.

Como esses estudos baseados exclusivamente em observações clínicas não estabelecem uma diferenciação entre o impacto psicológico das doenças em fase terminal e aquele decorrente de uma doença crônica, outros estudos mais controlados tentaram preencher esta lacuna.

A pesquisa de Waechter (1971), utilizando técnicas projetivas, e a de Spinetta, Rigler e Karon (1974), usando jogos estruturados, com o objetivo de obter respostas diretamente das crianças, sugerem que a criança terminal tem uma 
concepção mais amadurecida da morte do que seus pares que não experenciaram tal circunstância. É, provavelmente, o conhecimento de sua finitude que leva as crianças terminais, na pesquisa de Waechter, a se referirem mais freqüentemente ao temor da morte e da ameaça à integridade do corpo do que as crianças não terminais. Também na pesquisa de Spinetta e cols., é este mesmo amadurecimento que, segundo os autores, leva a criança terminal a um crescente sentimento de separação psicológica em relação ao hospital, pessoas e circunstâncias. Da mesma forma, os resultados da investigação de Brewster (1982) apontam para a importância da maturação dos processos cognitivos na compreensão da doença, e na concepção da morte como parte da doença.

No Brasil, existem poucas investigações sobre o conceito de morte, podendo- se citar, entre estas, os estudos de Torres $(1979,1993,1996)$, o de Fávero e Salim (1995) e o de Nunes, Carraro, Jou e Sperb (1998), as duas últimas utilizando-se da técnica de desenho na coleta de dados. Na primeira pesquisa de Torres (1979), a partir de uma perspectiva piagetiana, a avaliação quantitativa (teste de hipóteses) e os dados obtidos através do exame qualitativo através das respostas das crianças ao Instrumento de Sondagem do Conceito de Morte (Torres, 1979), permitiram identificar três níveis de desenvolvimento do conceito de morte, descritivos do pensamento da criança nos diferentes períodos de desenvolvimento cognitivo: Pré-Operacional, Operacional Concreto e Operacional Formal. Estes dados apontam, portanto, para a importância do nível de desenvolvimento cognitivo na evolução deste conceito, confirmando estudos precedentes (Anthony, 1972; Kane, 1978, 1979; Koocher, 1972), e para uma confirmação das formulações baseadas em Piaget, segundo as quais o conceito de morte se desenvolve em interação com outros conceitos como os de animado/inanimado, de tempo, de quantidade, de conservação/não conservação, causação e probabilidade (Fergunson, 1978; Kastembaum, 1959, 1983).

Considerando que esta primeira pesquisa investigou a relevância da variável cognitiva e lidou apenas com crianças de nível sócio-econômico médio/alto, uma segunda investigação foi realizada (Torres, 1993) com crianças de classe média-baixa, cujos resultados apontaram para a influência da variável sócio-econômica na aquisição do conceito de morte. Finalmente, uma terceira investigação foi desenvolvida com a finalidade de prosseguir o estudo da influência da variável sócio-econômica e cultural, desta vez, comparando-se o conceito de morte em crianças de três diferentes condições sócio-experienciais (Torres, 1996). Os resultados obtidos, reforçando os dados da pesquisa anterior, apontaram para uma defasagem cognitiva e uma inadequação do conceito de morte de crianças que vivem em condições de carência sócio-econômica e de marginalidade, quando comparadas com seus pares da classe média/alta. Esta inadequação ficou evidenciada no atraso da compreensão de algumas variáveis (extensão) e na aceleração da compreensão de outras (significado). Pode ser interpretada como um dos fatores de exacerbação do medo da morte que pode ser transformado e projetado sob a forma de atos agressivos.
De modo geral, quando se focaliza a violência, o comportamento anti-social das crianças que vivem em condição de carência sócio-econômica e de marginalidade, se estabelece uma relação direta, quase que mecânica, entre a miséria social e econômica e a violência. Os dados desta pesquisa reforçam a tese de que há outros fatores intermediando esta relação e, entre eles, a miséria cognitiva (Torres, 1996, 1999).

Tendo em vista que os estudos já realizados (Torres, 1979, 1993, 1996) focalizam o conceito de morte em função do nível cognitivo e avaliam o impacto da variável sócio-econômica e cultural, decidiu-se prosseguir nesta linha de investigação, considerando, ainda, a criança que experiencia uma condição rara e dolorosa - a doença crônica grave.

A finalidade da presente pesquisa é a de investigar a aquisição do conceito de morte em crianças portadoras de diferentes patologias, a fim de compará-las com crianças sadias de mesma condição sócio-econômica e mesmo nível cognitivo.

\section{Objetivos}

Os objetivos do estudo foram:

1) Verificar a relação entre desenvolvimento cognitivo e a evolução do conceito de morte em crianças portadoras de doenças crônicas;

2) Comparar a evolução do conceito de morte entre crianças portadoras de doenças crônicas e crianças sadias, com o mesmo nível sócio-econômico (carência) e mesmo nível cognitivo.

\section{Hipóteses}

$\mathrm{H}_{1}$. Crianças portadoras de doenças crônicas, de diferentes níveis de desenvolvimento cognitivo, avaliadas por tarefas piagetianas, diferem significativamente quanto à compreensão do conceito de morte nas dimensões extensão, significado, duração, tal como avaliadas pelo Instrumento de Sondagem do Conceito de Morte.

$\mathrm{H}_{2}$. Crianças portadoras de doenças crônicas e crianças sadias, do mesmo nível de desenvolvimento cognitivo, avaliadas por tarefas piagetianas, e de mesmo nível de carência sócio-econômica, diferem significativamente quanto à compreensão do conceito de morte nas dimensões extensão, significado e duração, tal como avaliadas pelo Instrumento de Sondagem do Conceito de Morte.

\section{Método}

\section{Sujeitos}

Cento e sessenta e sete crianças de ambos os sexos, entre 5 e 13 anos e 11 meses de idade, cuja escolaridade estende-se da Classe de Alfabetização a $8^{\mathrm{a}}$ série, foram selecionadas acidentalmente para o presente estudo entre crianças em condição de carência sócio-econômica, residentes, em sua maioria, em bairros afastados da zona norte ou centro da 
cidade, atendidas em hospitais da rede pública do Rio de Janeiro e portadoras de diversas patologias crônicas: câncer- incluindo leucemia (48); outras doenças hematológicas (81); doenças renais (4); HIV positivo (34). Nenhuma das crianças havia sofrido perdas significativas - pai, mãe ou irmãos.

\section{Instrumentos}

\section{Tarefas Piagetianas para a Avaliação do Nível de Desenvolvimento Cognitivo}

O nível de desenvolvimento cognitivo foi avaliado por tarefas em moldes piagetianos já selecionadas para os estudos precedentes (Torres, 1979, 1993, 1996).

Para testar a capacidade de conservação foram utilizados: teste de conservação da correspondência termo a termo e teste de conservação da quantidade; para avaliar a capacidade de acompanhar a forma de um argumento abstraído de seu conteúdo específico foram utilizados: teste de silogismo e problema de seriação de três termos, apresentado sob forma de proposições verbais.

Para determinação do nível de desenvolvimento cognitivo foram adotados critérios de classificação baseados nas sugestões de Phillips (1969/1971): a) classificar no subperíodo pré-operacional crianças que falhassem em uma ou nas duas tarefas de conservação; b) classificar no subperíodo de operações concretas, crianças que acertassem as duas tarefas de conservação e errassem as demais; e c) classificar no período de operações formais aquelas crianças que acertassem as quatro tarefas.

\section{Instrumento de Sondagem do Conceito de Morte}

O Instrumento de Sondagem do Conceito de Morte (Torres, 1979), consta de 36 itens planejados para avaliar as três dimensões - Extensão (universalidade), Significado (nãofuncionalidade), e Duração (irreversibilidade) - do conceito de morte biológica. Os itens pertinentes a cada uma das dimensões são precedidos de estórias-estímulo e são de três tipos: itens que exigem respostas apenas em termos de sim ou não, itens que exigem respostas em termos de sim ou não, seguidas de justificativa e itens totalmente abertos.

Os critérios de avaliação permitem a inclusão das respostas, para cada dimensão estudada, em três amplas categorias: ignorância ou aparente ignorância, noção restrita e noção completa. A atribuição de pontos varia de 0 a 3, havendo alguns itens relativos às dimensões de significado e duração que só admitem atribuição de valor 0 a 1 .

\section{Procedimento}

A aplicação dos instrumentos nas crianças portadoras de doenças crônicas foi realizada nos próprios hospitais, (enfermarias ou ambulatórios), após a avaliação e aprovação do Projeto de Pesquisa pela Comissão de Ética de cada instituição. As crianças a serem testadas eram convidadas, após o aplicador haver obtido o consentimento do acompanhante através de um termo de Consentimento Livre e Informado. Os instrumentos foram aplicados individualmente e verbalmente por um grupo de bolsistas de Iniciação Científica (CEPG/PIBIC) ou de Aperfeiçoamento (CNPq), treinados e supervisionados pela coordenadora do projeto. A testagem era iniciada, dizendo-se à criança que o aplicador gostaria de sua ajuda respondendo a algumas perguntas para um estudo ou trabalho de pesquisa. A seguir, eram aplicadas as tarefas para avaliação do nível cognitivo e finalmente o Instrumento de Sondagem do Conceito de Morte.

A avaliação das tarefas de desenvolvimento cognitivo classificou os 167 sujeitos testados em três grupos, segundo os períodos de desenvolvimento cognitivo. Tendo em vista que somente duas crianças foram classificadas no período Operacional Formal, as mesmas foram retiradas do teste de hipóteses, o que reduziu os participantes de 167 para 165 crianças.

Os escores do Instrumento de Sondagem do Conceito de Morte foram atribuídos aos 165 sujeitos agrupados em dois subperíodos de desenvolvimento cognitivo, segundo os critérios de avaliação acima descritos.

A relação entre o nível de desenvolvimento cognitivo e a evolução do conceito de morte em crianças portadoras de doenças crônicas $\left(\mathrm{H}_{1}\right)$ e a comparação entre crianças portadoras de doenças crônicas e crianças sadias do mesmo nível de desenvolvimento cognitivo e do mesmo nível de carência sócio-econômica $\left(\mathrm{H}_{2}\right)$ foram testadas através de Análises da Variância Simples, considerando-se cada dimensão - Extensão, Significado e Duração - em separado.

Para a comparação de crianças portadoras de doenças crônicas com crianças sadias $\left(\mathrm{H}_{2}\right)$ utilizou-se, no caso das crianças sadias, as crianças em condição de carência sócioeconômica já investigadas no estudo precedente (1996) ${ }^{3}$.

Finalmente, as respostas das crianças portadoras de doenças crônicas ao Instrumento de Sondagem do Conceito de Morte foram analisadas e comparadas com as das sadias, a fim de fornecer informações sobre as respostas típicas das crianças de cada nível cognitivo em cada uma das condições - carentes sadias e carentes portadoras de doenças crônicas.

\section{Resultados}

Nível de Desenvolvimento Cognitivo das Crianças Portadoras de Doenças Crônicas e das Crianças Sadias em Condição de Carência Sócio-Econômica.

A Tabela 1 apresenta a distribuição dos sujeitos, de ambos os grupos, por período de desenvolvimento cognitivo. A concentração de crianças no subperíodo Pré-Operacional, nos dois grupos, indica uma defasagem cognitiva dessas crianças em relação a seus pares de classe média/alta (Torres,

3 Esta amostra foi constituída de 142 crianças de ambos os sexos, entre 6 e 13 anos e 11 meses de idade, cuja escolaridade ia do C.A. à $4^{\text {a }}$ séria do $1^{\circ} \mathrm{Grau}$, alunos de uma escola pública do bairro de Acari, zona norte do Rio de Janeiro. Nenhuma das crianças havia sofrido perdas significativas - pai, mãe, ou irmãos. 
Tabela 1. Distribuição dos Grupos de Crianças Portadoras de Doenças Crônicas e de Crianças Sadias por Período de Desenvolvimento Cognitivo

\begin{tabular}{lccc}
\hline \multicolumn{3}{c}{ Períodos de Desenvolvimento Cognitivo } & \\
\hline & $\begin{array}{c}\text { Subperíodo } \\
\text { Pré Operacional }\end{array}$ & $\begin{array}{c}\text { Subperíodo } \\
\text { Operacional Concreto }\end{array}$ & Total \\
\hline $\begin{array}{l}\text { Crianças Portadoras de } \\
\text { Doenças Crônicas }\end{array}$ & 108 & 57 & 165 \\
\hline Crianças Sadias & 93 & 49 & 142 \\
\hline
\end{tabular}

1979, 1996).

Relação entre Nível de Desenvolvimento Cognitivo e Conceito de Morte em Crianças Portadoras de Doenças Crônicas.

\section{Hipótese 1:}

O sumário das Análises de Variância Simples para as três dimensões - Extensão, Significado e Duração - nos dois níveis cognitivos (Pré-Operacional e Operacional Concreto) das crianças portadoras de doenças crônicas é apresentado na Tabela 2.

Os valores de $\mathrm{F}=82,240, \operatorname{com} p=, 000 ; \mathrm{F}=61,711$, $\operatorname{com} p=, 000$ e $\mathrm{F}=57,734$, com $p=, 000$, para as três dimensões, Extensão, Significado e Duração, respectivamente, permitem concluir que as médias das crianças do subperíodo de Operações Concretas (Extensão 25,11; Significado 33,12; e Duração 7,74) são significativamente mais altas do que as médias das crianças do subperíodo PréOperacional (Extensão 17,74; Significado 21,1; e Duração 4,71) nas três dimensões do conceito de morte.

Comparação da Evolução do Conceito de Morte nas Três Dimensões Extensão, Significado e Duração entre Crianças Portadoras de Doenças Crônicas e Crianças Sadias de Mesmo Nível Cognitivo.

\section{Hipótese 2:}

Tendo em vista que no subperíodo Pré-Operacional a média de idade cronológica das crianças sadias $(9,0)$ é significativamente mais alta do que a das crianças portadoras de doenças crônicas $(7,7)$, com $p=, 02$, a comparação do

Tabela 2. Sumário das Análises da Variância Simples para as Dimensões Extensão, Significado, Duração nos Níveis Cognitivos Pré-Operacional e Operacional Concreto das Crianças Portadoras de Doenças Crônicas:

\begin{tabular}{llrrrrl}
\hline Dim. & Fonte & GL & SQ & MQ & F & SIG \\
\hline Ext. & Entre grupos & 1 & 2079,860 & 2079,860 & 82,240 &, 000 \\
\hline & Intra grupos & 163 & 4122,285 & 25,290 & - & - \\
\hline & Total & 164 & 6202,145 & - & - & - \\
\hline Signif. & Entre grupos & 1 & 5292,085 & 5292,085 & 61,711 &, 000 \\
\hline & Intra grupos & 163 & 13978,242 & 85,756 & - & - \\
\hline & Total & 164 & 19270,327 & - & - & - \\
\hline Dur. & Entre grupos & 1 & 341,149 & 341,149 & 57,734 &, 000 \\
\hline & Intra grupos & 163 & 963,154 & 5,909 & - & - \\
\hline & Total & 164 & 1304,303 & - & - & - \\
\hline
\end{tabular}

$\mathrm{GL}=$ Grau de Liberdade, $\mathrm{SQ}=$ Soma dos Quadrados, $\mathrm{MQ}=$ Média dos Quadrados, F= Razão F, SIG=Nível de Significância
Tabela 3. Sumário das Análises da Variância Simples para as Três Dimensões - Extensão, Significado e Duração - Considerando-se Crianças Sadias e Crianças Portadoras de Doenças Crônicas da Faixa Etária 6/8anos e Nível Cognitivo Pré-Operacional.

\begin{tabular}{llrrrrl}
\hline Dim. & \multicolumn{1}{c}{ Fonte } & GL & SQ & MQ & F & SIG \\
\hline Ext. & Entre grupos & 1 & 25,885 & 25,885 & 1,344 &, 249 \\
\hline & Intra grupos & 95 & 1829,229 & 19,255 & - & - \\
\hline & Total & 96 & 1855,113 & - & - & - \\
\hline Signif. & Entre grupos & 1 & 829,610 & 829,610 & 11,336 &, 001 \\
\hline & Intra grupos & 95 & 6952,164 & 73,181 & - & - \\
\hline & Total & 96 & & - & - & - \\
\hline Dur. & Entre grupos & 1 & 77,887 & 77,887 & 10,654 &, 002 \\
\hline & Intra grupos & 95 & 694,484 & 7,310 & - & - \\
\hline & Total & 96 & 772,371 & - & - & - \\
\hline
\end{tabular}

$\mathrm{GL}=$ Grau de Liberdade, $\mathrm{SQ}=$ Soma dos Quadrados, $\mathrm{MQ}=$ Media dos Quadrados, F= Razão F, SIG= Nível de Significância.

conceito de morte entre crianças destes dois grupos requereu que se agrupasse crianças da mesma faixa etária, 6 a 8 anos, a fim de controlar, até certo ponto, a influência desta variável. Os resultados das Análises da Variância Simples para este sub-período, são apresentados na Tabela 3.

No que se refere à dimensão Extensão, as médias de crianças sadias $(17,88)$ e de crianças portadoras de doenças crônicas $(16,85)$ não são significativamente diferentes, $\mathrm{F}=$ $1,344, \operatorname{com} p=, 249$. No que se refere à dimensão Significado $(25,12$ para as crianças sadias e 19,26 para as crianças portadoras de doenças crônicas) e Duração (6,49 para crianças sadias e 4,70 para as portadoras de doenças crônicas), as médias são significativamente diferentes, conforme os valores de $\mathrm{F}=11,336, \operatorname{com} p=, 001$ e $\mathrm{F}=10,654, \operatorname{com} p=, 002$, respectivamente.

Como no sub-período Operacional Concreto a média de idade cronológica das crianças sadias $(11,0)$ não difere significativamente da média de crianças portadoras de doenças crônicas $(10,6)$, com $p=95$, o controle da variável idade neste sub-período não foi necessário. Os resultados das Análises da Variância Simples para este subperíodo são encontrados na Tabela 4.

$\mathrm{O}$ valor de $\mathrm{F}=12,324, \operatorname{com} p=, 001$ indica que a média das crianças doentes $(25,11)$ é significativamente mais alta

Tabela 4. Sumário das Análises da Variância Simples para as Três Dimensões - Extensão, Significado e Duração - Considerando-se Crianças Sadias e Crianças Portadoras de Doenças Crônicas do Subperíodo Operacional Concreto.

\begin{tabular}{llrrrrl}
\hline Dim. & \multicolumn{1}{c}{ Fonte } & GL & \multicolumn{1}{c}{ SQ } & MQ & F & SIG \\
\hline Ext. & Entre grupos & 1 & 311,843 & 311,843 & 12,324 &, 001 \\
\hline & Intra grupos & 103 & $2.606,348$ & 25,304 & - & - \\
\hline & Total & 104 & $2.918,190$ & - & - & - \\
\hline Signif. & Entre grupos & 1 &, 895 &, 895 &, 039 &, 845 \\
\hline & Intra grupos & 103 & $2.392,953$ & 23,233 & - & - \\
\hline & Total & 104 & $2.393,848$ & - & - & - \\
\hline Dur. & Entre grupos & 1 & 14,587 & 14,587 & 5,535 &, 021 \\
\hline & Intra grupos & 102 & 268,797 & 2,635 & - & - \\
\hline & Total & 103 & 283,385 & - & - & - \\
\hline
\end{tabular}

$\mathrm{GL}=$ Grau de Liberdade, $\mathrm{SQ}=$ Soma dos Quadrados, $\mathrm{MQ}=$ Media dos Quadrados, F= Razão F, SIG= Nível de Significância. 
do que a de crianças sadias $(21,44)$ na dimensão Extensão. No que se refere às dimensões Significado (crianças doentes 33,12 e sadias 33,06), $\mathrm{F}=, 039, \operatorname{com} p=, 845$ e Duração ( crianças doentes 7,74 e sadias 8,48), $\mathrm{F}=5,535, \operatorname{com} p=$ ,021, os valores não são significativos.

Tendo em vista as diferentes patologias que constituem o grupo de crianças portadoras de doenças crônicas, cálculos a posteriori foram realizados a fim de avaliar as diferenças entre as médias obtidas pelas crianças de cada uma destas patologias nas três dimensões do conceito de morte.

Os dados obtidos nas Análises da Variância revelaram diferenças significativas entre as médias nas dimensões Extensão $(\mathrm{F}=2,609, p=, 055)$ e Significado $(\mathrm{F}=4,366, p=$ ,006) para o subperíodo Pré-Operacional, não sendo encontradas diferenças no subperíodo Operacional Concreto. Nas comparações das patologias consideradas por pares, através de testes de diferenças mínimas significativas, as únicas diferenças significativas ocorreram em relação às doenças hematológicas. Crianças portadoras deste tipo de enfermidade no subperíodo pré-operacional apresentam médias significativamente mais altas do que as demais enfermidades.

Como as médias para crianças de diferentes patologias no subperíodo de operações concretas não foram significativamente diferentes, testes a posteriori das mínimas diferenças foram desnecessários.

\section{Análise das Respostas aos Itens do Instrumento de Sondagem do Conceito de Morte}

A comparação da freqüência das respostas das crianças portadoras de doenças crônicas, para cada item do Instrumento de Sondagem do Conceito de Morte, com a das crianças sadias forneceu algumas informações sobre as respostas mais características de cada grupo.

No que se refere à compreensão da dimensão Extensão do conceito de morte (universalidade), conforme esperado, observa-se uma evolução gradual do nível Pré-Operacional para o nível Operacional Concreto nos dois grupos, ainda que algumas irregularidades possam ser assinaladas. No nível Pré-Operacional, embora em ambos os grupos predominem as respostas de noção restrita, no caso das crianças doentes, a freqüência das respostas de ignorância ou aparente ignorância é bem maior do que nas crianças sadias. Já no nível Operacional Concreto, a maior inconsistência se observa nas crianças sadias nas quais, embora predominando as respostas de noção restrita seguidas das de noção completa, observa-se um resíduo de respostas de ignorância em uma freqüência quase equivalente à noção completa. Em relação às crianças doentes, neste nível Operacional Concreto, predominam as respostas de noção restrita seguidas das respostas de noção completa. As de ignorância ou aparente ignorância praticamente desaparecem, indicando assim uma evolução mais regular, próxima a das crianças de nível sócio-econômico médio/alto (Torres, 1996).

4 As tabelas de freqüência são encontradas em Anexo do Relatório Técnico de Pesquisa enviado ao CNPq: NEPT, dez, 1999.
Quanto à compreensão da dimensão Significado da morte (não-funcionalidade), embora se observe, da mesma forma, uma evolução do nível Pré-Operacional para o Operacional Concreto, algumas peculiaridades podem ser observadas quando se compara crianças carentes sadias e crianças portadoras de doenças crônicas. No nível Pré-Operacional, nestes dois grupos, as respostas de ignorância ou aparente ignorância surgem com uma freqüência quase equivalente a das respostas indicadoras de noção completa, o que aponta para uma certa inconsistência quanto à compreensão do significado da morte, neste subperíodo cognitivo. No caso das crianças doentes, observa-se uma maior dificuldade para os itens que implicam na compreensão da deterioração do corpo ("os vermes comem os mortos ou não?" ou "com o passar do tempo o corpo dos mortos se desmancha ou não?") em relação aos quais predominam de forma absoluta as respostas de ignorância ou aparente ignorância, não ocorrendo praticamente respostas indicadoras de noção completa.

No que se refere ao significado da morte em termos de personificação, avaliada por itens especialmente elaborados para esta sondagem ("a morte mora em algum lugar?" ou "a morte é alguém que vem para levar as pessoas?”) ocorre de forma bastante freqüente nos dois grupos, neste nível PréOperacional.

Com relação aos itens que fornecem explicações mais abstratas do significado da morte ("o que é a morte?"ou "o que é estar morto?") a resposta predominante para as duas amostras foi "não sei", sendo muito difícil para as crianças deste subperíodo qualquer outro tipo de resposta.

No que se refere ao nível Operacional Concreto, embora haja aspectos semelhantes entre os dois grupos - crianças carentes sadias e carentes doentes - há também algumas peculiaridades que podem ser observadas. Em ambos predominam as respostas indicativas de noção completa do significado da morte, sendo que no caso das crianças sadias, estas respostas surgem apenas em relação àqueles itens que enfocam aspectos perceptivos mais óbvios ("os mortos abrem e fecham os olhos?", os mortos respiram?" ou "como você sabe se alguém está dormindo ou está morto?”). Já no que concerne aos itens que enfocam a deterioração do corpo ("os vermes comem o corpo dos mortos ou não?") as dificuldades persistem nestas crianças sadias, predominando respostas de ignorância ou aparente ignorância ao lado das respostas indicadoras de noção restrita, enquanto que nas crianças doentes predominam respostas indicadoras de noção completa, amplas que envolvem generalizações e/ ou explicações biologicamente essenciais. Da mesma forma, enquanto que nas crianças sadias persistem as respostas de personificação da morte, mesmo neste nível Operacional Concreto, nas crianças doentes a personificação ocorre com uma freqüência mínima. Quanto aos itens que envolvem a compreensão dos aspectos mais abstratos da morte a dificuldade persiste em ambos os grupos.

Quanto à compreensão da dimensão Duração, as crianças carentes sadias revelam um alto nível de compreensão da irreversibilidade da morte já no nível Pré-Operacional, evidenciado pelas respostas indicadoras de noção completa 
para a quase totalidade dos itens. Já as crianças portadoras de doenças crônicas apresentam neste nível Pré-Operacional maior ambivalência, ocorrendo respostas indicadoras de ignorância ou aparente ignorância e respostas indicadoras de noção completa em uma freqüência quase equivalente para vários itens. Chama ainda atenção neste grupo, as altas freqüências das respostas de ignorância em relação aos itens que implicam em aceitar "tratamento" para o morto ("daria sangue para a pessoa morta?" ou "daria remédio para a pessoa morta?") Para o único item que exigia justificativa de resposta ("mandaria enterrar a pessoa morta?" - "por quê?") as respostas foram do tipo parcial em termos de hábito ou tradição cultural.

Nas crianças portadoras de doenças crônicas, a compreensão da irreversibilidade da morte se deu de forma clara no nível Operacional Concreto (à exceção do item "doaria sangue a pessoa morta?" onde ainda persiste alguma dificuldade).

\section{Discussão}

No que se refere à avaliação do nível de desenvolvimento cognitivo, os dados revelaram uma defasagem cognitiva das crianças portadoras de doenças crônicas em relação a seus pares de classe média/alta (Torres, 1979, 1996) já encontrada em crianças carentes sadias (Torres, 1996). Como a comparação das distribuições desses dois grupos - crianças portadoras de doenças crônicas e crianças sadias - não revelou diferenças significativas e como os dois grupos se situam na mesma condição sócio-econômica, os dados parecem indicar que a doença crônica por si só não agrava a defasagem cognitiva já encontrada nas crianças sadias em condição de carência. Reforçam os dados obtidos na literatura e assinalados por Torres (1996), indicando que a condição sócio-econômica exerce uma influência sobre a psicogênese no que concerne ao desenvolvimento cognitivo.

Em relação à evolução do conceito de morte, os resultados, tal como hipotetizado, confirmam que há uma relação entre a evolução deste conceito e o nível de desenvolvimento cognitivo. Da mesma forma como ocorreu nos estudos precedentes (Torres, 1979, 1996), as crianças portadoras de doenças crônicas do subperíodo Operacional Concreto revelaram médias significativamente mais altas do que as crianças do subperíodo Pré-Operacional nas três dimensões do conceito de morte. Estes dados reforçam os achados da literatura sobre a relevância do nível cognitivo na evolução do conceito de morte e apontam para a importância das operações concretas como crucial na aquisição das três dimensões - Extensão, Significado e Duração - deste conceito.

Ao se introduzir a variável condição de doença crônica, comparando-se crianças do mesmo nível cognitivo (e mesma faixa etária no caso das crianças pré-operacionais) e diferentes condições - sadias e doentes - os dados obtidos sugerem um impacto desestruturante da doença sobre a aquisição do conceito de morte no nível Pré-Operacional. As crianças portadoras de doenças crônicas neste nível, obtiveram médias significativamente mais baixas do que as crianças sadias nas dimensões Significado e Duração, embora não se observe uma diferença significativa entre esses dois grupos - sadias e doentes - no que se refere à dimensão Extensão. Este impacto da doença no nível Pré-Operacional é confirmado, sobretudo, na avaliação qualitativa, em relação à dimensão Extensão, uma vez que as respostas de ignorância e/ ou aparente ignorância são mais freqüentes nestas crianças doentes do que nas crianças sadias para a maioria dos itens. Mesmo quando as respostas são indicativas de noção restrita, as explicações contrariam o esperado. Assim, para os itens "homem morre?" e "criança morre?" além das crianças doentes apresentarem muito mais respostas de ignorância ou aparente ignorância do que as sadias, a indicação de doença como causa específica da morte é muito menos freqüente nestas crianças do que nas crianças sadias.

O impacto da doença crônica no nível Pré-Operacional surge também na análise da dimensão Duração. Embora as crianças carentes sadias tenham a compreensão da irreversibilidade da morte já neste nível, as doentes se apresentam confusas e ambivalentes.

É possível que estes aspectos desestruturantes observados no nível Pré-Operacional se expliquem por ser provavelmente neste nível que a doença crônica tenha se instalado (ou sido diagnosticada) na maioria das crianças investigadas. Assim, o impacto do diagnóstico e do tratamento - envolvendo procedimentos altamente aversivos, numerosas consultas ambulatoriais ou mesmo hospitalizações, e portanto, afetando a rotina da criança, sua vida pessoal, familiar e escolar - por si só funcione como um fator de desestruturação e, mais que isso, determine o surgimento de mecanismos de defesa que vão bloquear ou desacelerar a aquisição do conceito de morte desta criança nesta fase. Aliás, a análise da comparação entre as diferentes patologias, consideradas duas a duas, parece reforçar esta interpretação. Embora não se possa descartar a possibilidade de algum viés, na amostragem, o fato é que nesta análise observa-se que nas crianças do nível Pré-Operacional portadoras de doenças crônicas hematológicas - possivelmente percebidas como menos ameaçadoras ou aparentemente menos ameaçadoras - o impacto do diagnóstico parece ser menor pois estas crianças obtiveram resultados significativamente mais altos do que as crianças com Aids e com câncer na dimensão Extensão, significativamente mais altos do que crianças com câncer na dimensão Significado e significativamente mais altos do que crianças com Aids na dimensão Duração.

Quando as crianças portadoras de doenças crônicas atingem o subperíodo das Operações Concretas, a situação se modifica totalmente e os dados sugerem que a doença - uma vez passada a fase inicial desestruturante - irá funcionar como um fator de amadurecimento do conceito de morte.

$\mathrm{Na}$ fase Operacional Concreta, a única diferença encontrada entre os dois grupos - crianças sadias e doentes - foi justamente em relação à dimensão Extensão, na qual as crianças doentes obtiveram escores significativamente mais altos do que as crianças sadias. Ora, a compreensão da universalidade da morte requer que a criança estabeleça uma relação entre morte e humanidade como uma categoria na qual ela mesma esteja incluída, e, portanto, no reconheci- 
mento da própria mortalidade. $\mathrm{O}$ fato das crianças portadoras de doenças crônicas, na fase Operacional Concreta, apresentarem melhores resultados do que as crianças sadias quanto à compreensão desta dimensão sugere que uma vez superado o bloqueio em função das defesas, a criança doente, no confronto com o sofrimento e a ameaça de morte, está pronta para estabelecer esta relação.

$\mathrm{O}$ amadurecimento do conceito de morte nas crianças portadoras de doenças crônicas pode ser ainda observado nos resultados relativos à dimensão Significado da morte. Embora não haja diferenças significativas nas médias obtidas por crianças doentes e crianças sadias, alguns dados na avaliação qualitativa chamam a atenção. Assim, por exemplo, enquanto permanecem as dificuldades das crianças sadias em relação a alguns itens que envolvem a deterioração do corpo, nas crianças doentes, as explicações predominantes para tais itens são amplas, lógica e biologicamente essenciais. Da mesma forma, também as respostas que envolvem personificação da morte que persistem nas crianças sadias mesmo no nível operacional concreto, nas crianças doentes surgem em uma freqüência muito inferior. Este dado parece apontar para o fato de que a personificação da morte utilizada como uma forma de defesa para eludir a morte, tão típica das crianças carentes e marginalizadas (Torres, 1996), tende a desaparecer nas crianças doentes.

Finalmente, a compreensão da irreversibilidade da morte está plenamente concluída nesta fase, e as diferenças quanto ao impacto das diferentes patologias na aquisição do conceito de morte, observadas na fase Pré-Operacional, também não mais se observam no subperíodo de Operações Concretas.

Este estudo permite concluir que: 1) a defasagem cognitiva encontrada em crianças em situação de carência sócioeconômica, tal como evidenciada na literatura, não parece ser agravada quando a carência vem associada à doença crônica; 2 ) há uma relação entre o conceito de morte e o nível de desenvolvimento cognitivo tal como encontrada na literatura, incluindo os estudos realizados com outras crianças brasileiras (Torres, 1979, 1996); 3) a doença crônica e o tipo de doença surgem como um fator importante na desorganização inicial e na lentificação da aquisição do conceito de morte na fase Pré-Operacional; 4) Na fase Operacional Concreta, o confronto com o sofrimento e a ameaça de morte surge como fator de amadurecimento na aquisição das três dimensões do conceito de morte. Estes dados parecem reforçar os achados de observações clínicas que sugerem que crianças gravemente doentes e/ou em fase terminal têm nítido conhecimento de sua condição (Bluebond-Langner, 1978; Raimbault, 1977/1979; Wright, 1974); e finalmente, 5) a comparação dos resultados obtidos nesta pesquisa com os estudos precedentes (Torres, 1979, 1996) sugere que o conceito de morte de crianças portadoras de doenças crônicas, na fase Operacional Concreta, se aproxima mais do de crianças sadias de nível sócio-econômico médio/alto do que de crianças sadias em condição de carência.

Em síntese, o abandono e a desassistência, tal como enfatizado por Torres (1996), parecem exercer um efeito ain- da mais devastador do que a doença. Na medida em que crianças portadoras de doenças crônicas são crianças fisicamente cuidadas e assistidas, quer pela equipe de saúde, quer pela família, não vivem em condição de abandono muitas vezes vivenciada pela criança carente sadia. Não obstante, $o$ risco do "abandono emocional" existe em face da dificuldade do adulto para falar com a criança acerca de sua condição. Desta forma, para que a "maturidade" da criança, conquistada às custas da doença e do sofrimento, não se perca, é necessário que os adultos se preparem para ajudá-la: preparando a criança para a hospitalização, as visitas ao médico, os procedimentos; discutindo com a criança seus problemas e questões que ela expressa verbalmente; antecipando e expressando para a criança aquelas preocupações que ela indica no comportamento mas não expressa verbalmente.

\section{Referências}

Adams, D.W. (1979). Childhood malignancy: the psychological care of the child and his family. Springfield, IL.: Charles C. Thomas.

Anthony, S. (1972). The discovery of death in childhood and after. New York, NY.: Basic Books.

Bibace, R. \& Walsh, M.E. (1980). Development of children's conceptions of illness. Pediatrics, 66, 912-917.

Bluebond-Langner, M. (1974). I know, do you? A study of awareness, communication and coping in terminally ill children. Em: Schoenberg, B.; Carr, A.C.; Kutscher, A.H.; Peretz, D. \& Goldberg, I. (Orgs.), Antecipating grief. (pp.171-181). New York, NY.: Columbia University Press.

Bluebond-Langner, M. (1978). The private worlds of dying children. New Jersey: Princeton University Press.

Bolduc, J. (1972). A developmental study of the relationship between experiences of death and age and development of the concept of death. Doctoral Dissertation, Columbia University, Ann Arbor, Michigan.

Brewster, A.B. (1982). Cronically ill hospitalized children's concepts of their illness. Pediatrics, 69, 355-362.

Cotton, C.R. \& Range, L. (1990). Children's death concepts: relationship to cognitive functioning, age, experience with death, fear of death and hopelessness. Journal of Clinical Child Psychology, 19, 123-127.

Fávero, M.H. \& Salim, C.M.R. (1995). A relação entre os conceitos de saúde, doença e morte: Utilização do desenho na coleta de dados. Psicologia: Teoria e Pesquisa, 11, 181-191.

Fergunson, F. (1978). Children cognitive discovery of death. Journal of the Association for the Care of Children in Hospitals, 7 (1),8-14.

Gartley, W. \& Bernasconi, M. (1967). The concept of death in children. Journal of Genetic Psychology, 110,71- 85.

Hornblum, J.N. (1978). Death concepts in childhood and their relationship to concepts of time and conservation. Doctoral Dissertation, Temple University.

Kalmbach, C.A. (1978). The relationship between the cognitive level of the child and his/her conception of death. Doctoral Dissertation, Florida State University, Florida.

Kane, B. (1978). A piagetian discussion of the development of children's concept of death. Em: Annual Piagetian Theory and 
the Helping Professions Conference, Proceedings Seventh Interdisciplinary Conference, Los Angeles (1), 65-72.

Kane, B. (1979). Children's concepts of death. Journal of. Genetic Psychology, 134,141-153.

Kastenbaum, R. (1959). Time and death in adolescence. Em Feifel, H. (org.), The meaning of death (pp.99-113).New York, NY.: Macgraw-Hill.

Kastenbaum, R. \& Aisenberg, R. (1983). A psicologia da morte. (A.P. Lessa, Trad.) São Paulo: Pioneira. (original publicado em 1976)

Koocher, G.P. (1972). Childhood death and cognitive development. $\mathrm{PhD}$, University of Missouri, Missouri.

Lonetto, R.(1980). Children's conceptions of death. N Y : Springer.

McIntire, M. S.; Angle, C.R.; Struempler, L.J. (1972). The concept of death in midwestern children and youth. American Journal of Diseases of Children, 123, 527-532.

Morrisey, J.R. (1963). Children's adaptations to fatal illness. Social Work, 8, 81-88.

Nagy, M.H. (1959). The child's view of death. Em Feifel, H. (org.), The meaning of death (pp. 79-98). New York: McGraw-Hill.

Natterson, J. \& Knundson, A. (1960). Children and their mothers: observations concerning fear of death in fatally ill. Psychosomatic Medicine, 22, 456-465.

Nunes, D.C., Carraro, L., Jou, G.I. \& Sperb, T. (1998). As crianças e o conceito de morte. Psicologia: Reflexão e Crítica, 11, 579590.

Orbach, I. \& Glaubman, H. (1978). Suicidal, agressive and normal children's perception of personal and impersonal death. Journal of Clinical Psychology, 34,850-857.

Orbach, I. \& Glaubman, H. (1979). The concept of death and suicidal behavior in young children. Journal of the American Academy of Child Psychiatry, 18, 668-678.

Peck, R. (1966). The development of concept of death in select male children. Doctoral Dissertation, New York University, NY.

Phillips, J.L. Jr. (1971). Origens do intelecto: A teoria de Piaget. (A. Cretella, Trad.). São Paulo: Nacional/USP (original publicado em 1969).

Raimbault, G. (1979). A criança e a morte. (R. C. Lacerda, trad.) Rio de Janeiro: Francisco Alves. (original publicado em 1977)

Reilly, T.P. , Hasazi, Y.E. \& Bond, L.A. (1983). Children's conceptions of death and personal mortality. Journal of Pediatric Psychology, 8(1), 21-31.

Safier, G.A. (1964). Study in relationships between the life and death concepts in children. Journal of Genetic Psychology, 105, 283-294.

Schilder, P. \& Wechsler, D. (1934). The attitude of children towards death. Journal of Genetic Psychology, 45, 406-451.

Schonfeld, D.J. \& Smilansky, S. (1989). A cross-cultural comparison of israeli and american children's death concepts. Death Studies, 13, 593-604.

Shaffer, D. \& Fisher, P. (1981). The epidemiology of suicide in children and young adolescents. Journal of the American Academy of Child Psychiatry, 20, 545-565.

Speece, M.W. \& Brent, S. (1984). Children's understanding of death: a review of three components of death concept. Child Development, 55, 1671-1686.
Spinetta, J., Rigler, D.J. \& Karon, M. (1974). Personal space : a measure of dying child's sense of isolation. Journal of Consulting and Clinical Psychology, 42, 751-756.

Tallmer, M., Formanek, R. \& Tallmer, J. (1974). Factors influencing children's concepts of death. Journal of Clinical Child Psychology, 3(2), 17-19.

Torres, W. da C. (1979). O conceito de morte na criança. Arquivos Brasileiros de Psicologia, 31(4), 9-34.

Torres, W. da C. (1993). Estudo da relação entre conceito de morte, nível de desenvolvimento cognitivo e nível sócio-econômico. (Relatório de Pesquisa ao CNPq) Rio de Janeiro: Instituto de Psicologia da Universidade Federal do Rio de Janeiro.

Torres, W. da C (1996). O desenvolvimento cognitivo e a aquisição do conceito de morte em crianças de diferentes condições sócio-experênciais. Tese de Doutorado, Faculdade de Ciências Médicas, UNICAMP, Campinas.

Torres, W. da C. (1999). A criança diante da morte: desafios. São Paulo: Casa do Psicólogo.

Townley, K. \& Thornburg, K (1980). Maturation of the concept of death in elementary school children. Education Research Quarterly, 5, 17-24.

Waechter, E. (1971). Children's awareness of fatal illness. American Journal.of Nursing, 71, 1168-1172.

Weininger, O. (1979). Young children's concepts of dying and dead. Psychological Reports, 44, 395-407.

Wright, L. (1974)- An emotional support program for parental of dying children. Journal of Clinical Psychology, 3 (2), 37-8.

Yalom, I. (1980). Existencial psychoterapy. New York: Basic Books.

Zweig, A.R. (1976). Children's attitudes toward death. Doctoral Dissertation, Northwestern University, Illinois. 